\title{
Research of Emergency Information Resourse Organization Based on Metadata
}

\author{
Lu Yanxia \\ Dalian Nesusoft University of Information \\ Dalian, China \\ luyanxia@neusoft.edu.cn \\ Zhou Ling \\ Dalian Nesusoft University of Information \\ Dalian, China \\ zhouling@neusoft.edu.cn
}

\author{
Wu Di \\ Dalian Nesusoft University of Information \\ Dalian, China \\ wudi@neusoft.edu.cn
}

\begin{abstract}
Introducing metadata management mechanism to build emergency information resources, the paper establishes the data storage metadata, data exchange metadata and data display metadata, and integrates the data of E-government inside-outside network. Information integration based on metadata will solve the problem that distributed, heterogeneous emergency information integrate and share difficultly. The method is practiced and evidenced in the development of Dalian Emergency Management Development Platform. Practice shows that information organization and management methods based on metadata management can enhance the flexibility of data manipulation of emergency management decision support system, and expansibility, portability, easy maintenance and data consistency of heterogeneous system data.
\end{abstract}

KeyWords-Metadata; Emergency; Information Resource Organization; Manangement; E-government

\section{INTRODUCTION}

In recent years, a lot of unexpected events caused by natural or man-made causes have caused a great number of casualties and economic losses, which makes the research on the emergency management of unexpected events become a hot issue. Emergency decision information system construction is not only a few system integration, but also the integration of the integrated system. It is the integration of system and information resources and the coordination of various departments, and is a cross regional, cross industry thousands of existing emergency management information system to the overall coordination of the work of the complex system. It is facing a great challenge to measure, so the core of the construction of the emergency system is to achieve its "integration" and "synergy", to achieve a variety of systems and information resources to work efficiently.

Effective emergency management should have the basic requirements of responding quickly and effectively, and organization coordination, adapting to the dynamic changes of events. The core of the emergency management of public emergencies is the response decision under the specific constraints, while the scientific response decision needs the systematic knowledge and information. The premise of achieving these basic requirements is based on the completeness of information acquisition, the validity of information processing and the timeliness of information distribution. But in the reality of emergency information resources management, if in a series of management process of emergency and resource information collection, reporting, cleaning, integration, analysis, sharing, application, there is any deviation, it is likely to have a very big impact on the emergency treatment, and sometimes even greater disaster. At present, as a kind of resource, the emergency information is a common understanding, but it is faced with the problem of how to manage and organize the emergency information resources accurately and efficiently.

In this context, this paper set up the information organization method of collecting and acquiring the information, the data analysis, the organization and the information in the public emergency accidents decision information, based on the flexibility of metadata for data processing. The purpose is to integrate the information resources of the emergency system, and solve the problem of the mechanism and management inthe distributed heterogeneous emergency information, which is difficult to integrate and share. It also conducted to provide the necessary information or knowledge to the emergency management decision, assistant decision maker for emergency decision and improve the response time of the decision.

\section{METADATA FUNCTION AND STRUCTURE}

Metadata is often defined as data about data. But there are still some differences in the understanding of the concept of metadata, some scholars have given the concept of metadata for specific applications. For example, Meta Data Coalition defines the metadata for information description which is about the structure of data, the application of data manipulation and the description of the process[1]. Kimball etc define the metadata for information that is outside of the actual data itself in the 
data warehouse environment[2]. There is also the interpretation of metadata for the data about the data, or other information about information[3]. Metadata can be defined in a narrow sense as the data to describe the data, which is the data structure, data updating, data dependence, data quality, etc. It can also be generalized understood as not only to achieve description data, but also realize data conversion, operation and management. This is a description of the problem and the operation data from the two levels. Therefore, metadata can be understood as: the process of data, data management, and the application of the structure and meaning of the description of data, and the goal is to provide a comprehensive guide to data resources. In addition metadata has been well developed and applied in the field of data storage and data warehouse[4-7].

Combined with the characteristics of emergency management system, the main function of emergency management information system is summarized as follows

(1). Metadata can be used to integrate a wide variety of emergency related information.

(2). Metadata definition can help end users to understand the data stored in the system from the semantic layer.

(3). Metadata can support dynamic request.

(4). Metadata can provide high quality data.

(5). Metadata can improve the flexibility of the performance or the operation in the system.

(6). Metadata can support the application of a variety of development tools.

(7). Metadata can improve the intelligence of the system.

\section{ARCHITECTURE OF AN EMERGENCY INFORMATION MANAGEMENT PLATFORM BASED ON METADATA MANAGEMENT}

As the emergency management has become a hot research topic in recent years, there is no uniform standard for the definition and classification of emergency information resources at present, but many scholars put forward their views on the emergency information resources. Liao Siqin introduced the application of the stored metadata based on cloud storage in the government information resources in her paper[8]. Zhang Weiping from a macro point of view introduced emergency resources, including emergency material reserve, emergency plan, rescue team information, emergency information system, as well as the government and social forces, involving all aspects of emergency management [9]. Chen Qing researched on cross sectoral government information sharing negotiation mechanism based on
Metadata. Qiu Jiangnan researched on the General Metadata in emergency field.

This paper considers that the emergency information management should cover the whole process of emergency management, and use advanced information technology and processing method based on metadata, to do a series of processes of the emergency management and decision making information collection, processing, integration, transmission, storage, dissemination and using. So as to provide timely and accurate and efficient information service for emergency decision-making command staffs, functional departments and the public media, to provide support for the solution of unexpected events. A architecture of emergency information management platform based on metadata management is given below.

In the emergency system, the requirement of information quality mainly refers to the accuracy, reliability, timeliness and safety of emergency information. In the construction of information in China, valuing hardware and despising software, valuing system construction and despising resource management. So it caused that a large number of hardware and network resources wasted. Therefore, in the construction of emergency response system, we have to correct or change this kind of error tendency, strengthen the effective organization and integration of emergency information. And through planning, management, organization, control and other means, we manage human, material and financial resources which are related to sudden public events. To ensure that the appropriate emergency information resources information is presented to the appropriate emergency decision makers at the appropriate time in the happening of a sudden public event.

The information in the emergency information system has a character of wide variety of sources, content complex, diverse formats. The information includes the history of case information, geographic information, personnel distribution information, alarm information, plan information, scene information, scheduling resources, decision-making resources, public reports etc. So we should provide high quality information to meet individual needs.

Because of the difference between the application platform and the user's internal system structure environment, the emergency decision makers often face a physically dispersed, heterogeneous border emergency management environment, when incident happens. So we must solve the problem of data exchange in heterogeneous environment. Information organization generally includes information collection, processing, transmission, storage, exchange, sharing, application and archiving process, and those process require a management based on metadata. 


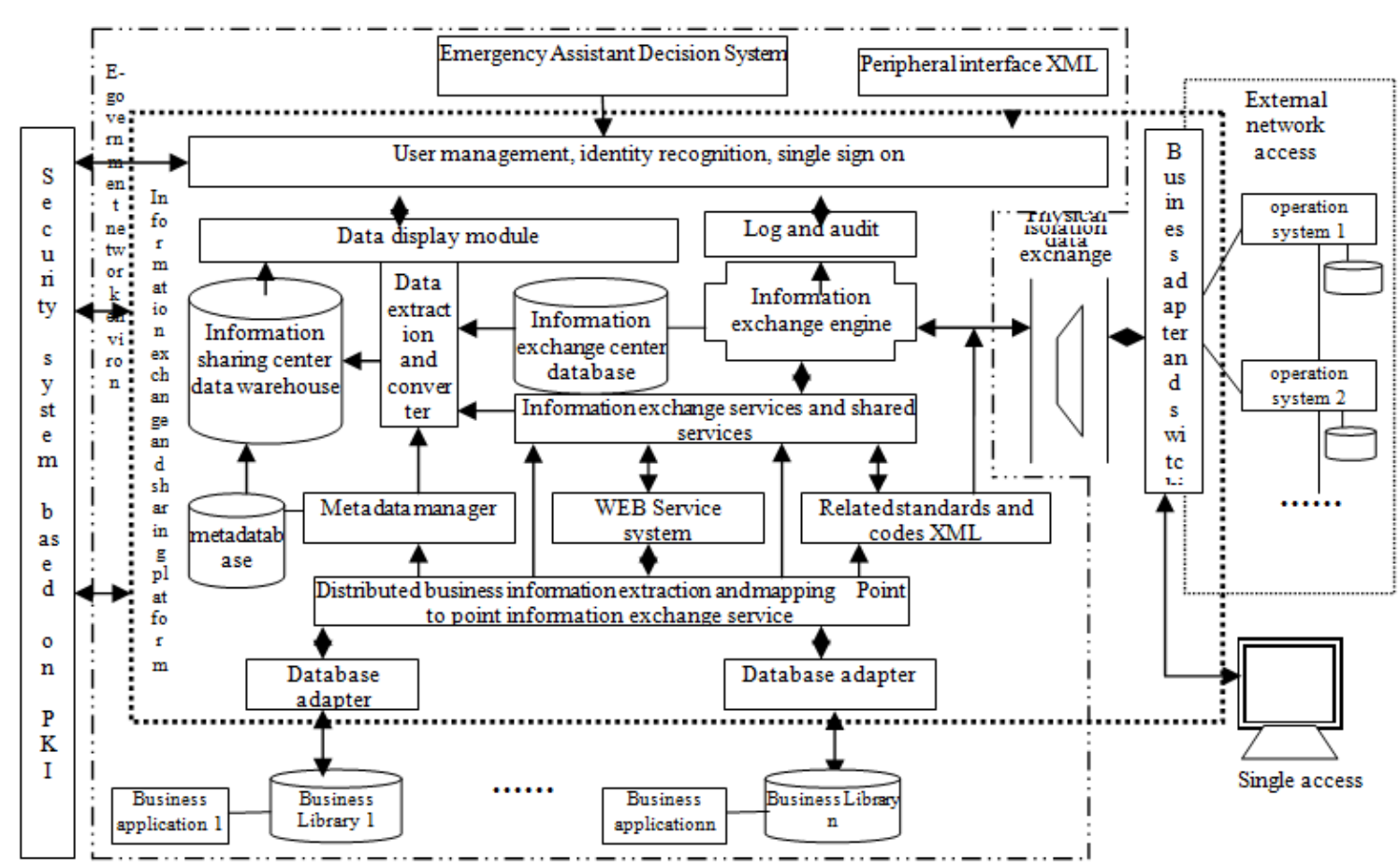

Figure 1. Architecture of an emergency information management platform based on Metadata

The data transmission and switching layer are mainly divided into two modules in this system. After the data which from an external business application system converted with exchange broker by a business adapter, it is sharing loaded by the internal and external network data exchange equipment under the condition of physical isolation. The data is automatically loaded into the Egovernment Intranet which with the network physical isolation. After automatically loaded, the external data is introduced into the information exchange center by the information exchange engine, and then loaded into the data warehouse of the information exchange platform through the data extraction and conversion. So the data can be further process and use. The data of each business application system of the electronic government affairs network is also loaded into the data warehouse, through the database adapter and distributed business information extraction and mapping and after data extraction and conversion. Data exchange module convert the organization form of heterogeneous data (such as DBF, TXT, etc.) into a system specific data form, which solves the problem of information integration of heterogeneous systems. If necessary, we can release the data of egovernment after exchange to the business application system or e-government extranet.

Its main functions include: the internal and external network data extraction and collection, data conversion and synchronization, metadata management, shared data interface. After the security of the legitimate, users can access through the data display module. The integration of information can be displayed through the data display module.

\section{STORAGE, DiSPLAY AND EXCHANGE OF INFORMATION BASED ON METADATA}

\section{A. Storage Metadata}

The information system achieves a function based on data, therefore flexible and effective management of data is the cornerstone of the system function. This platform uses metadata related to data management mechanism(Fig 2). For example, the entire structure of the database table is stored in an ordered array class. Each element in the array details detail all the relevant attribute information of the field, such as the relationship between the attributes, and the relationship between the tables, etc. Database table structure metadata can be described by $\mathrm{C}++$ as follows:

class CDbStr

CString name;
CString code;
CString fded;
WORD type;
int length;
CStringArray reltb;
$\ldots . .$.
\}...

CString name;

CString code;

CString fded;

WORD type

CStringArray reltb; 


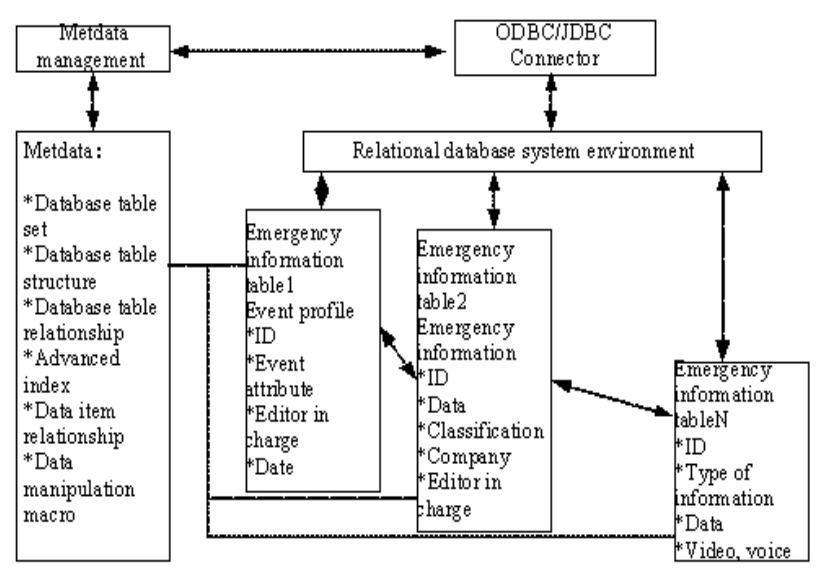

Figure 2. Data storage metadata management mechanism

\section{B. Data exchange Metadata}

As time goes on, the emergency management information system needs more and more information, not only the access to the information quantity and type is growing, but also the involving field is more extensive. So the data exchange and sharing between the systems will become more frequent and in-depth.

There is also a big difference between the data definition and the format in the system, beside system environment difference. Over time the range of the associated sets is also changing. The traditional way to modify the program code will bring a lot of work for the late development and maintenance. In this paper, provides a relatively simple and flexible way using metadata to solve the data exchange system. In the system, the mapping method of dynamic maintenance is introduced to express and manage the metadata of the system, it can be easily set and only need to maintain a simple mapping table can be achieved.

To solve the problem of data format or property between different systems, the system use visual interface which establish attribute correspondence. Before transferring data, application according to the previous mapping set needs to exchange data format transform through the corresponding conversion program. If there is a problem of data type mismatch, you can set up the window to complete the docking of the data field

\section{Data display Metadata}

The purpose of data exchange is to meet the needs of different users. In practice, the user can set the output mode of the data in accordance with their own style, such as the background, font format, etc. So this system is introduced to meet the user's individual input and output metadata. Figure 4 describes the process of extracting the metadata, for example, the following $\mathrm{C}++$ description is as follows:

\section{class Personalization \\ \{}

CString Pic;

CString Wav;

CString Pont;

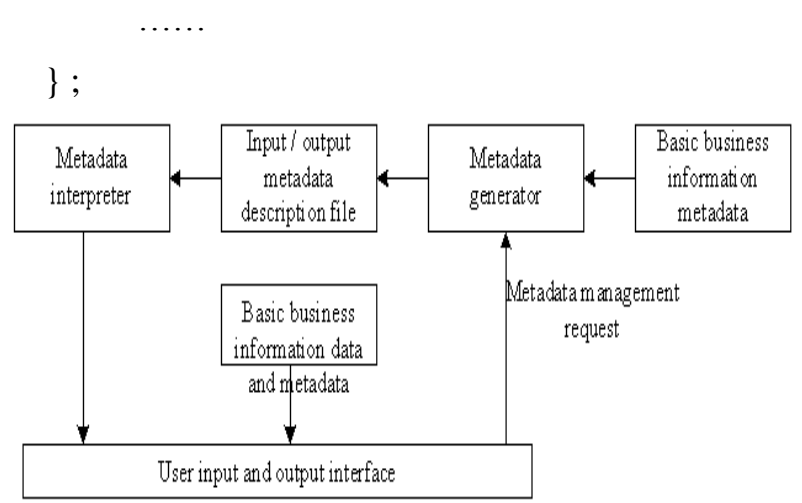

Figure 3.The implementation process of data display metadata

\section{CONCLUSIONS}

This paper discusses the application and implementation of metadata in the data storage, exchange and presentation of information exchange in the emergency management system from the point of view of system development practice. Related research achievements have been applied and practice in the egovernment construction project "Dalian government emergency command center application software system", and achieved good results.

\section{REFERENCES}

[1] Meta Data Coalition. Open Information Model [EB/OL]. http://xml.coverpages. org/mdc-oim.html 1999.

[2] KimballR, ReevesL, RossM,etal. The Data Warehouse Lifecycle Toolkit: ExpertMethods for Designing, Developing, and Deploying Data Warehouses [M]. New York: Wiley, 1998.

[3] Chen Ximing. Dublin Core analysis of [J]. metadata of Shanxi Library Journal, 2002, (3): 19-21.

[4] P.A.Bernstein. "Repositories and object-oriented databases". SIGMOD Record. 1998, 27(1):88-96.

[5] Lawrence A. West Jr., Traci J. Hess. "Metadata as a knowledge management tool: supporting intelligent agent and end user access to spatial data". Decision Support Systems, 2002, 32: 247- 264.

[6] Vassiliadis Panos, Bouzeghoub Mokrane, Quix Christoph. "Towards Quality-oriented Data Warehouse Usage and Evolution". Information Systems, 2000, 25(2):89-115.

[7] Vassiliadis Panos, Quix Christoph, Vassiliou Yannis, etc. "Data warehouse process management". Information Systems. 2001, 26(3): 205-236

[8] Zhou Yu, Liao Siqin, Hu Cuihong. Research on the preservation of government information resources based on cloud storage [J]. Journal of information resources, 2012,31 (4): 143-147.

[9] Zhang Weiping. Response to public emergencies requires the integration of government resources [J]. Journal of Guizhou Normal University (SOCIAL SCIENCE EDITION), 2007 (2): 1518.

[10] Chen hydrogen. Research on the information sharing and consultation system of the cross department government [J] information journal, 2014,33 (7): 188-193.

[11] Qiu Jiangnan, Liu Lili, Xu Jing, Wang Yanzhang. Research on the general standard of the emergency field of $[\mathrm{J}]$. information. 